\title{
PAEDIATRIC CONTRAST-ENHANCED ULTRASONOGRAPHY: A CHALLENGE WITH A PROMISING FUTURE
}

\author{
Éva KIS
}

Semmelweis University, Budapest, Hungary

Correspondence:

kiseva2009@gmail.com

Tel.: + 36205460477

Received: June 6, 2016; Accepted: June 12, 2016

Key words: Contrast-enhanced ultrasonography (CEUS) • Voiding urosonography (VUS) • Children.

In the last issue of Paediatrics Today two excellent well-articulated and focused reports were published. Ključevšek D. was the author of the review paper "Contrast-enhanced ultrasonography in children" and Cvitković Roić A, et al. of "Contrast-enhanced voiding urosonography (CeVus) as a diagnostic tool in evaluation of vesicoureteral reflux" $(1,2)$.

The appearance of ultrasound contrast agents (UCAs) opened up a new radiation free opportunity in sonography. Contrastenhanced ultrasonography (CEUS) is especially important in children who may in this way avoid radiation and sedation for selected indications. The introduction of dedicated contrast agent-specific software to the ultrasound equipment enables suppression of signals from the background tissue and visualisation of only the microbubble signals, without the fundamental grey-scale echoes. Today all major ultrasound vendors have contrast specific software available.

The report by Ključevšek (1) deals in detail with the major difficulty of pediatric application: UCAs in general have not been registered for individuals younger than 18 years of age. Although $70 \%$ of drugs commonly used in pediatrics are not licensed for use in children, off-label use of UCA makes the application questionable. However, in the last 15 years a large number of publications have appeared about childhood application, its advantages and pharmacological safety. Also, several independent professional societies have published guidelines primarily about voiding urosonography (VUS) based on literature reviews and prospective clinical trials (3). In the report there is excellent information, not only about intravesical application (voiding urosonogragraphy-VUS), but about the possibilities of intravenous application and indications for UCAs.

VUS has been used routinely in pediatrics in Europe since the late 1990s and to a lesser extent in Asia, Australia, and South America. Some months ago a significant step forward took place, when the U.S. Food and Drug Administration (FDA) approved Lumason from Bracco Diagnostics for characterizing focal liver lesions in adult and pediatric patients (4). This approval marks a major shift toward acceptance of CEUS in children and this may lead to the routine clinical usage of the method.

VUR is a common abnormality of the urinary tract in children. Diagnostic imaging of VUR is one of the most frequently used procedures in pediatric radiology departments all over the world. The main pediatric indication for CEUS is an intravesical injection for detecting vesicoureteric reflux. The procedure and grading of VUR with VUS is nowa- 
days well standardized. It is a more sensitive method for detection and grading of VUR than what was previously the gold standard, voiding cystourethrograhy (VCUG), and for this reason should be used in first place in the examination of VUR in childhood. Intravenous application of UCA is an increasingly widespread method used in adults. In children, the major barrier is off-label use, but there is hope for great progress in this matter. Only a few publications exist, about a small number of patients. The standardization of methods and the required dosage recommendations are missing.

In the liver the most important applications are the detection and characterization of focal liver lesions, assessment of blunt trauma, and evaluation for disseminated infections.

Investigation of transplanted livers with CEUS is much easier: it is better at imaging the hepatic artery, which is difficult in many cases, and discovering hyper vascularisation of one part of the liver is possible, even in the operating room. CEUS has advantages in blunt abdominal trauma (including the liver, spleen, kidneys, and pancreas), in exploration and characterization of focal lesions in the spleen, kidneys, and pancreas, and in evaluation of the bowel in children with inflammatory bowel disease. There are also possibilities to use CEUS in pleural or abdominal drainage procedures as well. Even though the FDA's current approval of Lumason in children is restricted to imaging of focal lesions of the liver, it is very likely that it will be followed by more widespread approval and will be extended to Sonovue, which is a similar agent well known in Europe.

There is no need for sedation for application of CEUS, unlike MRI, in small children. It is inexpensive compared to MRI or $\mathrm{CT}$, radiation free, can be used at the bed side or in the operating room, and it is possible to apply in patients with reduced renal func- tion, since it is eliminated through the lungs. It is important that only one serious anaphylactic reaction and only a few cases of minor adverse reactions have been detected in the large number of VUS studies in children. These were primarily the results of bladder catheterization.

In conclusion, currently, contrast-enhanced voiding urosonography is a radiationfree, sensitive, and safe imaging modality for vesicoureteral reflux in children. There are clear guidelines from professional societies about indications and applications in pediatric patients. The FDA's approval of Lumason from Bracco Diagnostics, for characterizing focal liver lesions in adult and pediatric patients was a great step taken some months ago. Intravenous application of UCAs has a broad indication. Unfortunately, it is still less commonly used in children nowadays. However, hopefully this will change: in cases of focal liver masses, abdominal trauma, transplanted liver, kidney, inflammatory bowel disease it could often replace CT and MR examinations.

Conflict of interest: The author declares that she has no conflict of interest.

\section{References}

1. Ključevšek D. Contrast-enhanced ultrasonography in children. Paediatrics Today. 2016;12(1):49-55.

2. Cvitković Roić A, Roić G, Palčić I, Jaklin Kekez A. Contrast-enhanced voiding urosonography (CeVus) as a diagnostic tool in evaluation of vesicoureteral reflux. Paediatrics Today. 2016;12(1):56-65.

3. Riccabona M, Vivier PH, Ntoulia A, Darge K, Avni F, Papadopoulou F, et al.; ESPR Uroradiology Task Force. ESPR uroradiology task force imaging recommendations in paediatric uroradiology, part VII: standardised terminology, impact of existing recommendations, and update on contrastenhanced ultrasound of the paediatric urogenital tract. Pediatr Radiol. 2014;44(11):1478-84.

4. Bracco Diagnostics Inc. [homepage on the Internet]. Bracco Diagnostics Inc. - 259 Prospect Plains Road., Building H, Monroe Township, NJ 08831. [cited 2016 Mayl 9]. Available from: http://imaging.bracco.com/us-en/lumason. 